REPRESENTATION THEORY

An Electronic Journal of the American Mathematical Society

Volume 3, Pages 416-434 (November 9, 1999)

S $1088-4165(99) 00085-0$

\title{
THE HOWE DUALITY \\ AND THE PROJECTIVE REPRESENTATIONS OF SYMMETRIC GROUPS
}

\author{
ALEXANDER SERGEEV
}

\begin{abstract}
The symmetric group $\mathfrak{S}_{k}$ possesses a nontrivial central extension, whose irreducible representations, different from the irreducible representations of $\mathfrak{S}_{k}$ itself, coincide with the irreducible representations of the algebra $\mathfrak{A}_{k}$ generated by indeterminates $\tau_{i, j}$ for $i \neq j, 1 \leq i, j \leq n$ subject to the relations

$$
\tau_{i, j}=-\tau_{j, i}, \quad \tau_{i, j}^{2}=1, \quad \tau_{i, j} \tau_{m, l}=-\tau_{m, l} \tau_{i, j} \text { if }\{i, j\} \cap\{m, l\}=\emptyset ;
$$$$
\tau_{i, j} \tau_{j, m} \tau_{i, j}=\tau_{j, m} \tau_{i, j} \tau_{j, m}=-\tau_{i, m} \text { for any } i, j, l, m .
$$

Recently M. Nazarov realized irreducible representations of $\mathfrak{A}_{k}$ and Young symmetrizers by means of the Howe duality between the Lie superalgebra $\mathfrak{q}(n)$ and the Hecke algebra $H_{k}=\mathfrak{S}_{k} \circ C l_{k}$, the semidirect product of $\mathfrak{S}_{k}$ with the Clifford algebra $C l_{k}$ on $k$ indeterminates.

Here I construct one more analog of Young symmetrizers in $H_{k}$ as well as the analogs of Specht modules for $\mathfrak{A}_{k}$ and $H_{k}$.
\end{abstract}

\section{Summary}

Lately, we have witnessed an increase of interest in the study of representations of symmetric groups. In particular, in their projective representations.

Recall that the symmetric group $\mathfrak{S}_{k}$ has a nontrivial central extension whose irreducible representations do not reduce to those of $\mathfrak{S}_{k}$ but coincide (may be identified) with the irreducible representations of the algebra $\mathfrak{A}_{k}$ determined by generators $\tau_{i, j}$ for $i \neq j, 1 \leq i, j \leq n$ subject to the relations

$$
\begin{aligned}
\tau_{i, j}=-\tau_{j, i}, \quad \tau_{i, j}^{2} & =1, \quad \tau_{i, j} \tau_{m, l}=-\tau_{m, l} \tau_{i, j} \text { if }\{i, j\} \cap\{m, l\}=\emptyset ; \\
\tau_{i, j} \tau_{j, m} \tau_{i, j} & =\tau_{j, m} \tau_{i, j} \tau_{j, m}=-\tau_{i, m} \text { for any } i, j, l, m .
\end{aligned}
$$

In [N1] Nazarov realized irreducible representations of $\mathfrak{A}_{k}$ by means of an orthogonal basis constructed in each of the spaces of the representations and indicating the action of the generators $\tau_{i, i+1}$ on them (an analog of the Young orthogonal form). In [N2], with the help of an "odd" analog of the degenerate affine Hecke algebra Nazarov constructed elements of the algebra $H_{k}=\mathfrak{S}_{k} \circ C l_{k}$, the semidirect product of $\mathfrak{S}_{k}$ with the Clifford algebra $C l_{k}$ on $k$ indeterminates. Certain elements of $H_{k}$ serve as analogs of Young symmetrizers.

Received by the editors September 4, 1998 and, in revised form, September 8, 1999.

1991 Mathematics Subject Classification. Primary 20C30, 20C25, 17A70.

Key words and phrases. Projective representations, symmetric group, Howe duality.

I am thankful to D. Leites for support; to him and the referee for help.

(C)1999 American Mathematical Society 
Here I construct one more analog of Young symmetrizers in $H_{k}$ as well as analogs of Specht modules (cf. [Ja]) for the algebras $\mathfrak{A}_{k}$ and $H_{k}$. This construction is based on another form of expression of Young symmetrizers based on the notion of seminormal representation (an analog of Gelfand-Tsetlin basis) for the symmetric group (see $[\mathrm{OV}],[\mathrm{R}],[\mathrm{Mu}]$ ) one can express Young symmetrizers in the form distinct from both the classical one (that of Weyl [W]) and that suggested in [Ju2]. Observe that the structure of Nazarov's symmetrizers is rather complicated; Jones simplified them [Jo1], [Jo2] but not completely. The symmetrizers constructed in this paper are simpler in structure. Our method is also applicable to the $q$-analogs of the Young symmetrizers of the usual and projective representations of $\mathfrak{S}_{k}$; cf. [JN].

This paper is an expanded and rewritten (thanks to a shrewd referee) version of my short note math.RT/9810148. The reader might also be interested in the results of [Ya1] and [Ya2], slightly related with this paper; namely, [Ya1] describes $\mathfrak{A}_{k}$ as a Howe dual of $\mathfrak{q}(n)$ and in [Ya2] a Howe dual of $\mathfrak{q}(m) \oplus \mathfrak{q}(n)$ is established to be the hyperoctahedral group.

1.1. For the general notion of a seminormal representation see $[R]$. In this section I will formulate it for $\mathfrak{S}_{k}$ in convenient terms (cf. [OV]).

Let $\mathfrak{S}_{k}$ be the symmetric group on $k$ symbols, $s_{i j} \in \mathfrak{S}_{k}$ the transposition. The Jucys-Murphy elements are defined as follows (cf. [Ju1], [Mu]):

$$
x_{1}=0, x_{2}=s_{12}, x_{3}=s_{13}+s_{23}, \ldots x_{k}=s_{1 k}+s_{2 k}+\cdots+s_{k-1, k} .
$$

As in [Ma], we will identify the partition $\lambda$ of a positive integer $k$ with a Young diagram of $k$ cells. A Young tableau of the form $\lambda$ is the Young diagram corresponding to $\lambda$ filled in with the integers 1 to $k$. The tableau is called a standard one if the numbers that fill it do not decrease along the rows left to right and along the columns downwards.

For any partition $\lambda$ denote by $\mathcal{T}(\lambda)$ the set of standard tableaux of shape $\lambda$. For $\Lambda \in \mathcal{T}(\lambda)$ and $1 \leq p \leq k$, we set $c_{\Lambda}(p)=j-i$, where $(i, j)$ is the slot in $\Lambda$ occupied by $p$.

1.2. Theorem $([\mathrm{Mu}],[\mathrm{OV}],[\mathrm{R}])$. Let $S^{\lambda}$ be the irreducible representation of $\mathfrak{S}_{k}$ corresponding to the partition $\lambda$. Then in $S^{\lambda}$ there exists a common eigenbasis for the Jucys-Murphy elements $x_{1}, \ldots, x_{k}$; the basis can be indexed with the elements of $\mathcal{T}(\lambda)$ as follows:

$$
x_{p} v_{\Lambda}=c_{\Lambda}(p) v_{\Lambda} \quad \text { for any } \Lambda \in \mathcal{T}(\lambda) \text { and } 1 \leq p \leq k .
$$

Let us partially order the pertitions with respect to dominance, i.e., we set

$$
\mu \geq \lambda \Longleftrightarrow \sum_{i=1}^{l} \mu_{i} \geq \sum_{i=1}^{l} \lambda_{i} \text { for } l=1,2, \ldots
$$

Let $\Lambda_{c}$ be a Young tableau of shape $\lambda$ consecutively filled in along columns (that is where the subscript comes from) from left to right. Set

$$
\kappa_{\Lambda_{c}}=\prod_{i=1}^{k}\left(j-x_{i}\right)
$$

where $j$ is the number of the column occupied by $i$. Since the elements $x_{1}, \ldots, x_{k}$ pairwise commute, the order of factors in (1.1.1) is irrelevant. 
Example 1. For

$$
\begin{array}{rlr}
1 & 4 & 7 \\
\Lambda_{c}= & 5 & 8 \\
3 & 6 &
\end{array}
$$

we have

$$
\kappa_{\Lambda_{c}}=1 \cdot\left(1-x_{2}\right)\left(1-x_{3}\right)\left(2-x_{4}\right)\left(2-x_{5}\right)\left(2-x_{6}\right)\left(3-x_{7}\right)\left(3-x_{8}\right) .
$$

1.3. Theorem. Let $S^{\mu}$ be the irreducible representation of $\mathfrak{S}_{k}$ corresponding to the partition $\mu$ of $k$. Then

i) $\kappa_{\Lambda_{c}}\left(S^{\mu}\right)=0$ if $\mu>\Lambda_{c}$;

ii) $\kappa_{\Lambda_{c}}\left(v_{\Lambda}\right)=0$ if $\Lambda_{c} \neq \Lambda$ for $\Lambda \in \mathcal{T}(\lambda)$ and the basis vectors $v_{\Lambda_{c}}$ as in Theorem 1.2;

iii) $\kappa_{\Lambda_{c}}\left(v_{\Lambda_{c}}\right)=1^{\lambda_{1}} 2^{\lambda_{2}} \ldots n^{\lambda_{n}} v_{\Lambda_{c}}$, where $\lambda_{1} \geq \lambda_{2} \geq \cdots \geq \lambda_{n}$ are nonzero integers of the partition $\lambda$.

Proof. Let $\mu \geq \lambda$ and $\Lambda$ be a standard tableau of shape $\mu$; let $v_{\Lambda} \in S^{\mu}$ be the corresponding vector for the seminormal basis (i.e., as in section 1.2). If $\Lambda \neq \Lambda_{c}$, then there exists a $j$ such that the $j$-th columns of the tableaux $\Lambda$ and $\Lambda_{c}$ are distinct whereas all the columns with lesser numbers are identical. Since the orders of the tableaux coincide and $\mu \geq \lambda$, it follows that $\mu_{j}^{\prime} \leq \lambda_{j}^{\prime}$ (these are the lengths of the $j$-th columns of the tableaux $\Lambda$ and $\Lambda_{c}$, respectively).

Let $p$ be the least of the numbers from the $j$-th column of $\Lambda$ (such a number exists because $\Lambda \neq \Lambda_{c}$ and $\mu_{j}^{\prime} \leq \lambda_{j}^{\prime}$ ). Since $\Lambda$ is standard, the slot that $p$ occupies is $(1, j+1)$. Hence,

$$
x_{p} v_{\Lambda}=c_{\Lambda}(p) v_{\Lambda}=(j+1-1) v_{s}=j v_{\Lambda}, \quad \text { i.e., }\left(j-x_{p}\right) v_{\Lambda}=0 .
$$

Since $j-x_{p}$ enters $\kappa_{\Lambda_{c}}$ as a factor, $\kappa_{\Lambda_{c}} v_{\Lambda}=0$. This proves i) and ii).

To prove iii), observe that

$$
\left(j-x_{p}\right) v_{\Lambda_{c}}=(j-(j-i)) v_{\Lambda_{c}}=i v_{\Lambda_{c}},
$$

where $i$ is the number of the row occupied by $x_{p}$. This proves iii).

1.4. Lemma. Let $p<k$ and let $\mathfrak{S}_{p}$ be considered as the subgroup of $\mathfrak{S}_{k}$ that preserves all but the first $p$ symbols. Let $U$ be a $\mathfrak{S}_{k}$-module, $\varepsilon$ the nontrivial onedimensional representation and $a=\sum_{\sigma \in \mathfrak{S}_{p}} \varepsilon(\sigma) \sigma$. Let $v \in U$ be a nonzero vector fixed by a transposition $s_{l k}$ for $l \leq p$. Then

$$
a\left(\sum_{i \in \mathfrak{S}_{p}} s_{i k}\right) v=a v .
$$

Proof. Let $P=\{1, \ldots, p\}$. Then

$$
\begin{aligned}
& a\left(\sum_{i \in P} s_{i k}\right) v=a\left(\sum_{i \in P} s_{i k}\right) s_{l k} v \\
& =a\left(1+\sum_{i \in P \backslash\{l\}} s_{i k} s_{k l}\right) v=a\left(1+\sum_{i \in P \backslash\{l\}} s_{l i} s_{i k}\right) v \\
& =a\left(1-\sum_{i \in P \backslash\{l\}} s_{i k}\right) v=a\left(2-\sum_{i \in P} s_{i k}\right) v ;
\end{aligned}
$$

hence, $a\left(\sum_{i \in P} s_{i k}\right) v=a v$. 
1.5. Corollary. Let $R_{\Lambda_{c}}$ and $C_{\Lambda_{c}}$ be the row and column stabilizers of the tableau $\Lambda_{c}$; let $U$ be $a \mathfrak{S}_{k}$-module and $v \in U$ a nonzero $R_{\Lambda_{c}}$-invariant vector. Set $a_{\Lambda_{c}}=$ $\sum_{\sigma \in C_{\Lambda_{c}}} \varepsilon(\sigma) \sigma$. Then $\kappa_{\Lambda_{c}} v=a_{\Lambda_{c}} v$.

Proof. Induction on the number of columns in $\Lambda_{c}$. If $\Lambda_{c}$ consist of one column, then by induction on $k$ we easily verify the identity

$$
\left(1-s_{12}\right)\left(1-s_{13}-s_{23}\right) \ldots\left(1-s_{1 k}-\cdots-s_{k-1, k}\right)=\sum_{\sigma \in \mathfrak{S}_{k}} \varepsilon(\sigma) \sigma .
$$

So for one column the statement is true.

Let tableau $\Lambda_{c}$ consist of $n$ columns $\Lambda_{1}, \ldots, \Lambda_{n}$; let $\Lambda_{c}^{*}$ be obtained from $\Lambda_{c}$ by deleting the last column. Then

$$
\begin{aligned}
\kappa_{\Lambda_{c}} v & =\kappa_{\Lambda_{c}^{*}} \prod_{i \in \Lambda_{n}}\left(n-x_{i}\right) v=\prod_{i \in \Lambda_{n}}\left(n-x_{i}\right) \cdot \kappa_{\Lambda_{c}^{*}} v \\
& =\text { (by induction) }=\prod_{i \in \Lambda_{n}}\left(n-x_{i}\right) \cdot a_{\Lambda_{c}^{*}} v=a_{\Lambda_{c}^{*}} \prod_{i \in \Lambda_{n}}\left(n-x_{i}\right) v .
\end{aligned}
$$

Let $i \in \Lambda_{n}$; then

$$
a_{\Lambda_{c}^{*}}\left(n-x_{i}\right) v=a_{\Lambda_{c}^{*}}\left(n-\sum_{\alpha \in \Lambda_{1}} s_{\alpha i}-\sum_{\alpha \in \Lambda_{2}} s_{\alpha i}-\cdots-\sum_{\alpha \in \Lambda_{n}, \alpha<i} s_{\alpha i}\right) v .
$$

By Lemma $1.4 a_{\Lambda_{c}^{*}}\left(\sum_{\alpha \in \Lambda_{j}} s_{\alpha i}\right) v=a_{\Lambda_{c}^{*}} v$ for $j=1, \ldots, n-1$, so $a_{\Lambda_{c}^{*}}\left(n-x_{i}\right) v=$ $a_{\Lambda_{c}^{*}}\left(1-\sum_{\alpha \in \Lambda_{n}, \alpha<i} s_{\alpha i}\right) v$. Hence, if $\Lambda_{n}=\{i, i+1, \ldots, i+l\}$, then

$$
\begin{aligned}
\kappa_{\Lambda_{c}} v & =a_{\Lambda_{c}^{*}}\left(1-s_{i, i+1}\right)\left(1-s_{i, i+2}-s_{i+1, i+2}\right) \ldots\left(1-s_{i, i+l}-\cdots-s_{i+l-1, i+l}\right) v \\
& =a_{\Lambda_{c}^{*}}\left(\sum_{\sigma \in \Lambda_{n}} \varepsilon(\sigma) \sigma\right) v=a_{\Lambda_{c}} v .
\end{aligned}
$$

Thus, if $v=\sum_{\sigma \in R_{\Lambda_{c}}} \sigma=b_{\Lambda_{c}}$, then

$$
e_{\Lambda_{c}}=a_{\Lambda_{c}} b_{\Lambda_{c}}=\kappa_{\Lambda_{c}} b_{\Lambda_{c}},
$$

i.e., we have expressed the Young symmetrizer $e_{\Lambda_{c}}$ as $\kappa_{\Lambda_{c}} b_{\Lambda_{c}}$. In $\S 3$ a similar construction will be used to construct projective analogs of Young symmetrizers.

\section{Auxiliary Data}

Let $\mathfrak{S}_{k}$ be the symmetric group, $C l_{k}$ the Clifford algebra generated by $k$ indeterminates $p_{1}, \ldots, p_{k}$ subject to the relations

$$
p_{i}^{2}=-1, p_{i} p_{j}+p_{j} p_{i}=0 \text { for } i \neq j .
$$

The symmetric group acts on $C l_{n}$ permuting the generators, so we can form a semidirect product $H_{k}=\mathfrak{S}_{k} \circ C l_{k}$, i.e., the linear span of the elements $f \sigma$, where $f \in C l_{k}$ and $\sigma \in \mathfrak{S}_{k}$ with the multiplication satisfying

$$
\sigma f=f^{\sigma} \cdot \sigma \text { (here } f^{\sigma} \text { is the result of the action of } \sigma \in \mathfrak{S}_{k} \text { on } f \in C l_{k} \text { ). }
$$

Set

$$
\tau_{i, j}=\frac{1}{\sqrt{2}}\left(p_{i}-p_{j}\right) s_{i, j}
$$


As is not difficult to verify, the relations (1.1) hold; hence, the algebra generated by the $\tau_{i, j}$ is isomorphic to $\mathfrak{A}_{n}$. Besides, $\mathfrak{A}_{n}$ supercommutes with $C l_{n}$; hence, $H_{n}=\mathfrak{A}_{n} \otimes C l_{n}$, as superalgebras if we define parity in $\mathfrak{A}_{n}$ by setting $p\left(\tau_{i, j}\right)=\overline{1}$.

Let $\mathbb{N}$ be the set of positive integers, $\overline{\mathbb{N}}$, another , "odd", copy of $\mathbb{N}$; its elements are barred. Let $V$ be a superspace of superdimension $(n, n)$ with the fixed basis $\left\{e_{i}\right\}_{i=1}^{n} \amalg\left\{e_{\bar{i}}\right\}_{i=\overline{1}}^{\bar{n}}$. Define the odd operator $Q: V \longrightarrow V$ by setting

$$
Q\left(e_{i}\right)=e_{\bar{i}} ; \quad Q\left(e_{\bar{i}}\right)=-e_{i} .
$$

The (super)centralizer of $Q$ in $\operatorname{Mat}(V)$ is denoted by $\mathrm{Q}(V)$; cf. [BL]. We denote the Lie superalgebras associated with the associative superalgebras Mat $(V)$ and $\mathrm{Q}(V)$ by $\mathfrak{g l}(V)$ and $\mathfrak{q}(V)$, respectively. We set $\mathfrak{q}(n)=\mathfrak{q}(V)$ for $n=\operatorname{dim} V$.

Select a basis in $\mathfrak{q}(n)$ : let the $e_{i}^{*}$ be the left dual basis to the $\left\{e_{i}: i=1, \ldots, n\right.$; $\overline{1}, \ldots, \bar{n}\} ;$ set

$$
E_{i, j}=e_{i} \otimes e_{j}^{*}+e_{\bar{i}} \otimes e_{\bar{j}}^{*} ; \quad F_{i, j}=e_{i} \otimes e_{\bar{j}}^{*}+e_{\bar{i}} \otimes e_{j}^{*} .
$$

Then $\mathfrak{h}=\operatorname{Span}\left(E_{i, i}\right.$ and $\left.F_{i, i}: i=1, \ldots, n\right)$ is a Cartan subalgebra in $\mathfrak{q}(n)$ and $\mathfrak{b}=\operatorname{Span}\left(E_{i, j}\right.$ and $\left.F_{i, j}: i \leq j\right)$ is a Borel subalgebra; cf. [Pe]. Set further $\mathfrak{n}_{-}=\operatorname{Span}\left(E_{i, j}\right.$ and $\left.F_{i, j}: i>j\right)$ and $\mathfrak{n}_{+}=\operatorname{Span}\left(E_{i, j}\right.$ and $\left.F_{i, j}: i<j\right)$. Then, clearly, $\mathfrak{q}(n)=\mathfrak{n}_{-} \oplus \mathfrak{h} \oplus \mathfrak{n}_{+} ;$any finite dimensional irreducible $\mathfrak{q}(n)$-module $U=U^{\lambda}$ is uniquely, up to an isomorphism, determined by its highest weight and the irreducible $\mathfrak{h}$-module $U^{+}$of highest weight vectors annihilated by $\mathfrak{n}_{+}$, and for any $u \in U^{+}$we have $h u=\lambda(h) u$ for any $h \in \mathfrak{h}_{\overline{0}}$; cf. [Pe]. Observe that since $\mathfrak{h}$ is, unlike the case of Lie algebras, not commutative, $U^{+}$is seldom one-dimensional; cf. [S3]. This module $U$ will be denoted by $U^{\lambda}$.

We will stick to the following terminology; $\mathrm{cf}[\mathrm{BL}],[\mathrm{L}]$. The representation of a superalgebra $A$ in the superspace $V$ is irreducible if it does not contain homogeneous (with respect to parity) subrepresentations distinct from 0 and $V$ itself.

Thus, there are two types of irreducible representations: those that do not contain any nontrivial subrepresentations (called of general type or of type $G$ ) and those that contain inhomogeneous invariant subspaces (call them of type $Q$ ). If $V$ is of finite dimension, then in the first case its centralizer, as of $A$-module, is isomorphic to $\mathfrak{g l}(1)$, whereas in the second case to $\mathfrak{q}(1)$.

Let $V_{1}$ and $V_{2}$ be finite dimensional irreducible modules over $A_{1}$ and $A_{2}$, respectively. Then $V_{1} \otimes V_{2}$ is an irreducible $A_{1} \otimes A_{2}$-module except for the case where both $V_{1}$ and $V_{2}$ are of type $Q$. In the latter case, the centralizer of the $A_{1} \otimes A_{2}$ module $V_{1} \otimes V_{2}$ is isomorphic to $C l_{2}$, the Clifford superalgebra with 2 generators. (For further interesting details see [Sch].)

If $e \in C l_{2}$ is a minimal idempotent, then $e\left(V_{1} \otimes V_{2}\right)$ is an irreducible $A_{1} \otimes A_{2^{-}}$ module of type $G$ that we will denote by $2^{-1}\left(V_{1} \otimes V_{2}\right)$.

Let $\mathbb{M}=\mathbb{N} \coprod \overline{\mathbb{N}}$ be ordered as follows:

$$
\overline{1}<1<\overline{2}<2<\overline{3}<3 \ldots
$$

We will call the elements from $\mathbb{N}$ "even" and those form $\overline{\mathbb{N}}$ "odd" ones; so we can encounter an "even" odd element, etc.

The partition $\lambda$ of $k$ is called a strict one if its parts are pairwise distinct. To a strict partition $\lambda$ we assign a shifted diagram (see [Ma]) obtained from the ordinary diagram by shifting its $i$-th row by $i-1$ cell to the right for $i=1,2, \ldots$. A shifted tableau of shape $\lambda$ is a filling of a shifted diagram of shape $\lambda$ with numbers from $\mathbb{M}$. The shifted tableau is called a standard one if: 
i) the numbers that fill it do not decrease from left to right along rows and downwards along columns;

ii) the symbols from $\mathbb{N}$ strictly increase along columns (downwards);

iii) the symbols from $\overline{\mathbb{N}}$ strictly increase along rows (from left to right).

For the strict partition $\lambda$ denote by $S T(\lambda)$ the set of strict standard tableaux of shape $\lambda$ filled with the numbers $1, \ldots, k$, where $k=\lambda_{1}+\lambda_{2}+\ldots$ If $\Lambda \in S T(\lambda)$ and $1 \leq p \leq k$, then set $c_{\Lambda}(p)=\sqrt{(j-i)(j-i+1)}$, where $(i, j)$ is the slot in $\Lambda$ occupied by $p$.

Denote by $l(\lambda)$ the number of nonzero parts of the partition $\lambda$ and set

$$
\delta(\lambda)= \begin{cases}0 & \text { if the number of nonzero parts of } \lambda \text { is even, } \\ 1 & \text { otherwise. }\end{cases}
$$

As is shown in [S1], the algebras $\mathfrak{q}(n)$ and $H_{k}$ constitute a Howe-dual pair in the superspace $W_{k}=V^{\otimes k}$. The $\mathfrak{q}(n)$-action is uniquely determined by the identity action in $V$ extended as a superdifferentiation onto the tensor algebra $T(V)=$ $\sum W_{k}$. The symmetric group $\mathfrak{S}_{k}$ acts on $W_{k}$ by the formula $\sum_{k \geq 0}$

$$
\begin{aligned}
& s_{i, i+1}\left(v_{1} \otimes \cdots \otimes v_{i} \otimes v_{i+1} \otimes \otimes \cdots \otimes v_{k}\right. \\
& \quad=(-1)^{p\left(v_{i}\right) p\left(v_{i+1}\right)} v_{1} \otimes \cdots \otimes v_{i+1} \otimes v_{i} \otimes \otimes \cdots \otimes v_{k},
\end{aligned}
$$

where $p\left(v_{i}\right)$ and $p\left(v_{i+1}\right)$ are parities of respective vectors. Recall that $\mathfrak{q}(n)$ is defined as the centralizer of an odd operator $Q$ whose square is -id. The element $p_{i} \in H_{k}$ acts in $W_{k}$ as the operator

$$
1 \otimes \cdots \otimes 1 \otimes Q \otimes 1 \otimes \cdots \otimes 1 \quad(Q \text { occupies the } i \text {-th slot }) .
$$

2.1. Lemma. Let $w \in W_{k-1}$, set $\pi_{k}=\sum_{\alpha<k} \tau_{\alpha k}$, and also

$$
E_{i m}^{(2)}=\sum_{j=1}^{n}\left(E_{i j} E_{j m}-F_{i j} F_{j m}\right), \quad F_{i m}^{(2)}=\sum_{j=1}^{n}\left(F_{i j} E_{j m}-E_{i j} F_{j m}\right) .
$$

Then

i) $\pi_{k}\left(w \otimes e_{i}\right)=\frac{1}{\sqrt{2}} \sum_{j=1}^{n}\left(F_{i j}-p_{k} E_{i j}\right)\left(w \otimes e_{j}\right)$;

ii) $\left.\left.\pi_{k}^{2}\left(w \otimes e_{i}\right)=\frac{1}{2} \sum_{m=1}^{n}\left(\left(E_{i m}^{(2)} w\right) \otimes e_{m}\right)+\left(F_{i m}^{(2)} w\right) \otimes e_{\bar{m}}\right)\right)+\sum_{m=1}^{n}\left(E_{m m} w\right) \otimes e_{i}$.

Proof. i) It suffices to assume that $w=e_{i_{1}} \otimes \cdots \otimes e_{i_{k-1}}$, where all the indices $i_{1}$, $\ldots, i_{k-1}$ are "even", i.e., belong to $\mathbb{N}$, not $\overline{\mathbb{N}}$. Then

$$
\begin{aligned}
\pi_{k}\left(w \otimes e_{i}\right)=\sum_{\alpha<k} \tau_{\alpha k}\left(w \otimes e_{i}\right) & =\frac{1}{\sqrt{2}} \sum_{\alpha<k}\left(p_{\alpha}-p_{k}\right) s_{\alpha k}\left(w \otimes e_{j}\right) \\
& =\frac{1}{\sqrt{2}} \sum_{\alpha<k}\left(p_{\alpha}-p_{k}\right) w_{\alpha}^{i} \otimes e_{i_{\alpha}}
\end{aligned}
$$

where $w_{\alpha}^{i}$ is obtained from $w=e_{i_{1}} \otimes \cdots \otimes e_{i_{k-1}}$ by replacing $e_{i_{\alpha}}$ with $e_{i}$. Hence,

$$
\pi_{k}\left(w \otimes e_{i}\right)=\frac{1}{\sqrt{2}} \sum_{j=1}^{n} \sum_{i_{\alpha}=j}\left(p_{\alpha}-p_{k}\right) w_{\alpha}^{i} \otimes e_{j} .
$$


But, on the other hand, $\sum_{i_{\alpha}=j} w_{\alpha}^{i}=E_{i j} w$ and $\sum_{i_{\alpha}=j} p_{\alpha} w_{\alpha}^{i}=F_{i j} w$ implying that

$$
\begin{aligned}
\pi_{k}(w & \left.\otimes e_{i}\right)=\frac{1}{\sqrt{2}} \sum_{j=1}^{n}\left(F_{i j} w \otimes e_{j}-E_{i j} w \otimes e_{\bar{j}}\right) \\
& =\frac{1}{\sqrt{2}} \sum_{j=1}^{n}\left(F_{i j}\left(w \otimes e_{j}\right)-w \otimes e_{\bar{i}}-p_{k} E_{i j}\left(w \otimes e_{\bar{i}}\right)+w \otimes e_{\bar{i}}\right) \\
& =\frac{1}{\sqrt{2}} \sum_{j=1}^{n}\left(F_{i j}-p_{k} E_{i j}\right)\left(w \otimes e_{j}\right)
\end{aligned}
$$

and heading i) is proven.

ii)

$$
\begin{aligned}
\pi_{k}^{2}(w & \left.\otimes e_{i}\right)=\pi_{k}\left(\frac{1}{\sqrt{2}} \sum_{j=1}^{n}\left(F_{i j}-p_{k} E_{i j}\right)\left(w \otimes e_{j}\right)\right) \\
& =-\frac{1}{\sqrt{2}} \sum_{j=1}^{n}\left(F_{i j}-p_{k} E_{i j}\right) \pi_{k}\left(w \otimes e_{j}\right) \\
& =-\frac{1}{2} \sum_{j=1}^{n}\left(F_{i j}-p_{k} E_{i j}\right) \sum_{m=1}^{n}\left(F_{j m}-p_{k} E_{j m}\right)\left(w \otimes e_{m}\right) \\
& =-\frac{1}{2} \sum_{j, m=1}^{n}\left(F_{i j}-p_{k} E_{i j}\right)\left(F_{j m}-p_{k} E_{j m}\right)\left(w \otimes e_{m}\right) \\
& =-\frac{1}{2} \sum_{j, m=1}^{n}\left(\left(F_{i j} F_{j m}-E_{i j} E_{j m}\right)-p_{k}\left(E_{i j} F_{j m}-F_{i j} E_{j m}\right)\right)\left(w \otimes e_{m}\right) \\
& =\frac{1}{2} \sum_{j, m=1}^{n}\left(\left(E_{i j} E_{j m}-F_{i j} F_{j m}\right)+p_{k}\left(E_{i j} F_{j m}-F_{i j} E_{j m}\right)\right)\left(w \otimes e_{m}\right) .
\end{aligned}
$$

Let us calculate the sum by parts. We have

$$
\begin{aligned}
\left(E_{i j} E_{j m}-F_{i j} F_{j m}\right)\left(w \otimes e_{m}\right) & \\
= & E_{i j}\left(E_{j m} w \otimes e_{m}+w \otimes e_{j}\right)-F_{i j}\left(F_{j m} w \otimes e_{m}+w \otimes e_{\bar{j}}\right) \\
= & E_{i j} E_{j m} w \otimes e_{m}+E_{j m} w \otimes E_{i j} e_{m}+E_{i j} w \otimes e_{j}+w \otimes e_{i} \\
& -F_{i j} F_{j m} w \otimes e_{m}+F_{j m} w \otimes F_{i j} e_{m}-F_{i j} w \otimes e_{\bar{j}}-w \otimes e_{i} \\
= & E_{i j} E_{j m} w \otimes e_{m}-F_{i j} F_{j m} w \otimes e_{m}+E_{m m} w \otimes e_{i} \\
& +F_{m m} w \otimes e_{\bar{i}}+E_{i j} w \otimes e_{j}-F_{i j} w \otimes e_{\bar{j}} .
\end{aligned}
$$

and

$$
\begin{aligned}
p_{k}\left(E_{i j}\right. & \left.F_{j m}-F_{i j} E_{j m}\right)\left(w \otimes e_{m}\right) \\
= & p_{k} E_{i j}\left(F_{j m} w \otimes e_{m}+w \otimes e_{\bar{j}}\right)-p_{k} F_{i j}\left(E_{j m} w \otimes e_{m}+w \otimes e_{j}\right) \\
= & -E_{i j} E_{j m} w \otimes e_{\bar{m}}-F_{m m} w \otimes e_{\bar{i}}-E_{i j} w \otimes e_{j}-w \otimes e_{i} \\
& +F_{i j} E_{j m} w \otimes e_{\bar{m}}+E_{m m} w \otimes e_{i}+F_{i j} w \otimes e_{\bar{j}}+w \otimes e_{i} \\
= & \left(F_{i j} E_{j m}-E_{i j} F_{j m}\right) w \otimes e_{\bar{m}}+E_{m m} w \otimes e_{i} \\
& +F_{i j} w \otimes e_{\bar{j}}-E_{i j} w \otimes e_{j}-F_{m m} w \otimes e_{\bar{i}} .
\end{aligned}
$$


Therefore,

$$
\begin{gathered}
\frac{1}{2} \sum_{j, m=1}^{n}\left(\left(E_{i j} E_{j m}-F_{i j} F_{j m}\right)+p_{k}\left(E_{i j} F_{j m}-F_{i j} E_{j m}\right)\right)\left(w \otimes e_{m}\right) \\
=\frac{1}{2} \sum_{j, m=1}^{n}\left(\left(E_{i j} E_{j m} w \otimes e_{m}-F_{i j} F_{j m} w \otimes e_{m}\right)\right. \\
\left.\quad+\left(F_{i j} E_{j m}-E_{i j} F_{j m}\right) w \otimes e_{\bar{m}}+2 E_{m m} w \otimes e_{i}\right) \\
=\frac{1}{2} \sum_{m=1}^{n}\left(E_{i m}^{(2)} w \otimes e_{m}+F_{i m}^{(2)} w \otimes e_{\bar{m}}\right)+\sum_{m=1}^{n} E_{m m} w \otimes e_{i} .
\end{gathered}
$$

2.2. For the convenience of the reader we formulate the main result of [S1] in the following suitable form. Observe that if $\lambda$ is a strict partition of $k$ and $\lambda_{n+1}=0$, then $\lambda$ can be interpreted as a linear functional on $\mathfrak{h}_{\overline{0}}$ : set

$$
\lambda\left(E_{i i}\right)=\lambda_{i} .
$$

Theorem. The superalgebras $H_{k}$ and $\mathfrak{q}(n)$ are mutual centralizers in the superspace $W_{k}$ and the following decomposition takes place:

$$
W_{k}=\bigoplus_{\lambda} 2^{-\delta(\lambda)} T^{\lambda} \otimes V^{\lambda},
$$

where $\lambda$ runs over strict partitions of $k$ such that $\lambda_{n+1}=0$; here $T^{\lambda}$ is an irreducible (in the super sence) $H_{k}$-module corresponding to $\lambda$, and $V^{\lambda}$ an irrreducible $\mathfrak{q}(n)$ module.

If $\delta(\lambda)=0$, then the corresponding representation $T^{\lambda}$ is of type $G$, it is of type $Q$ if $\delta(\lambda)=1$.

The dimension of the space (superstructure ignored) of the highest weight vectors $\left(V^{\lambda}\right)^{+}$in $V^{\lambda}$ is equal to $2^{(l(\lambda)+\delta(\lambda)) / 2}$ copies of $T^{\lambda}$.

2.3. Corollary. The $H_{k}$-module of highest weight vectors in $W_{k}$ is a free $\mathrm{Cl}_{k}$ module.

Proof. By Theorem $W_{k}^{+}=\bigoplus_{\lambda} 2^{-\delta(\lambda)} T^{\lambda} \otimes\left(V^{\lambda}\right)^{+}$, so the $H_{k}$-module of highest weight vectors in $W_{k}$ is equal to $2^{-\delta(\lambda)} T^{\lambda} \otimes\left(V^{\lambda}\right)^{+}$. By considering the dimension of $\left(V^{\lambda}\right)^{+}$and taking into account the type $(G$ or $Q)$ of its irreducibility we get the statement desired.

2.4. Let us denote the $H_{k}$-module of highest weight vectors of weight $\lambda$ in $W_{k}$ by $R^{\lambda}$.

Lemma. Let $\mathfrak{g}=\mathfrak{q}(n)$; let $\mathfrak{b}$ and $\mathfrak{h}$ be defined as above and $V$ a $\mathfrak{g}$-module on which $\mathfrak{h}_{\overline{0}}$ acts diagonally. Let $\left(V^{\lambda}\right)^{+}$be the set of $\mathfrak{b}$-highest vectors of weight $\lambda$.

If $u \in U(\mathfrak{g})$ and $u\left(\left(V^{\lambda}\right)^{+}\right) \subset\left(V^{\lambda}\right)^{+}$, then there exists $w \in U(\mathfrak{h})$ such that $\left.u\right|_{\left(V^{\lambda}\right)^{+}}=\left.w\right|_{\left(V^{\lambda}\right)^{+}}$.

Proof. Let $u=\sum u_{\alpha}$ be the weight decomposition of $u \in U(\mathfrak{g})$ with respect to $\mathfrak{h}_{\overline{0}}$. Observe that $\left(V^{\lambda}\right)^{+}$is the $\lambda$ weight space of $V$. Therefore, if $v \in V_{\lambda}$, then $u(v)=\sum_{\alpha} u_{\alpha} v$ and, if $u_{\alpha} v \neq 0$, then the weight of $u_{\alpha} v$ is equal to $\lambda+\alpha$. Thus, thanks to the condition $u(v) \in\left(V^{\lambda}\right)^{+}$, we may assume that $u=u_{0} \in U(\mathfrak{g})^{\mathfrak{h}} \overline{\overline{0}}$, where $U(\mathfrak{g})^{\mathfrak{h} \overline{\bar{o}}}$ is the centralizer of $\mathfrak{h}_{\overline{0}}$. 
Further, thanks to [S2], we know that $U(\mathfrak{g})^{\mathfrak{h}_{\overline{0}}} \cong U(\mathfrak{h}) \oplus L$, where $L=U(\mathfrak{g})^{\mathfrak{h}_{\overline{0}}} \cap$ $U(\mathfrak{g}) \mathfrak{b}^{+}$and where $\mathfrak{b}^{+}$is the linear span of the positive roots in $\mathfrak{b}$, is a two-sided ideal in $U(\mathfrak{g})^{\mathfrak{h} \bar{o}}$.

Hence, $u=w+u_{1}$, where $w \in U(\mathfrak{h})$ and $u_{1} \in L$; this implies that $u v=w v$ for $v \in\left(V^{\lambda}\right)^{+}$.

Let $T$ be a finite dimensional $H_{k}$-module. The $\mathfrak{q}(n)$-module $U$ corresponding to it via Howe duality is determined by the formula

$$
U=\operatorname{Hom}_{H_{k}}\left(T, W_{k}\right) \text { for } W_{k}=V^{\otimes k} .
$$

It is not difficult to deduce from Theorem 2.2 that likewise $T$ is uniquely recoverable from $U$, namely,

$$
T=\operatorname{Hom}_{\mathfrak{q}(n)}\left(U, W_{k}\right) .
$$

Further on, since $U$ is a $\mathfrak{q}(n)$-submodule of $W_{k}$, it follows that all its weights with respect to $\mathfrak{h}_{\overline{0}}$ are of the form $m_{1} \varepsilon_{1}+\cdots+m_{n} \varepsilon_{n}$, where the $\varepsilon_{i}$ are the weights of the standard $\mathfrak{q}(n)$-module $V$ and the $m_{i}$ are nonnegative integers. This implies that the formal character of the $\mathfrak{q}(n)$-module $U$ equal to $\sum m_{\chi} e^{\chi}$ (here $\chi$ runs over the set of weights of $U$ with respect to $\left.\mathfrak{h}_{\overline{0}}\right)$ is a polynomial in $x_{1}=e^{\varepsilon_{1}}, \ldots, x_{n}=e^{\varepsilon_{n}}$. This polynomial is called the characteristic of $T$.

Example 2. Consider the Clifford superalgebra $C l_{k}$ as a module over $H_{k}\left(\mathfrak{S}_{k}\right.$ acts by permuting the generators of $C l_{k}$ while $C l_{k}$ acts on itself via the left regular representation). The corresponding characteristic $q_{k}$ is the coefficient of $t^{k}$ in the decomposition

$$
\frac{\prod_{i=1}^{n}\left(1+x_{i} t\right)}{\prod_{i=1}^{n}\left(1-x_{i} t\right)}=\sum_{k \geq 0} q_{k}(x) t^{k}
$$

In [S1] I showed that the $x$-character of $T^{\lambda}$ for the strict partition $\lambda$ is equal to $2^{(l(\lambda)-\delta(\lambda)) / 2} Q_{\lambda}(x)$, where $Q_{\lambda}(x)$ is the projective Schur function (for its definition see [Ma]). Therefore, the characteristic of $R^{\lambda}$ is equal precisely to $Q_{\lambda}(x)$.

2.5. Set $P_{\lambda}(x)=2^{-l(\lambda)} Q_{\lambda}(x)$. Denote by $S S T(\lambda)$ the set of standard tableaux of arbitrary shape with elements from $\mathbb{M}$ and such that

a) scanning the tableau from left to right and downwards the last of the symbols encountered should be an "even" one;

b) for every $i=1,2, \ldots$ the total number of occurrences of any of the symbols $i$ or $\bar{i}$ is equal to $\lambda_{i}$.

Lemma. Let $\lambda$ be a strict partition of $k$ and $\lambda_{1}>\cdots>\lambda_{n}>0$. Then

$$
P_{\lambda_{1}}(x) \ldots P_{\lambda_{n}}(x)=\sum_{\mu} m_{\lambda \mu} P_{\mu}(x),
$$

where the sum runs over strict partitions $\mu$ such that $\mu \geq \lambda$ (with respect to dominance partial order) and $m_{\lambda \mu}$ is equal to the number of tableaux from $S S T(\lambda)$ of shape $\mu$.

Proof of this statement is an easy corollary of the multiplication table for projective Schur functions; cf. [P], $\S 4$. 
2.6. Even and odd Jucys-Murphy elements (cf. [N2]). Set

$$
x_{1}=0, x_{2}=s_{12}\left(1+p_{1} p_{2}\right), \ldots, x_{k}=\sum_{i<k} s_{i k}\left(1+p_{i} p_{k}\right) .
$$

The odd analogs of the Jucys-Murphy elements $\pi_{i}$ are defined as

$$
\pi_{1}=0, \pi_{2}=\tau_{12}, \ldots, \pi_{k}=\sum_{i<k} \tau_{i k} .
$$

Observe that $\pi_{i}=-\frac{1}{\sqrt{2}} p_{i} x_{i}$ and the distinction of $\pi_{i}$ from $x_{i}$ is that the former supercommute with the elements of the Clifford superalgebra $C l_{k}$.

The following statement proven by M. Nazarov in [N2] (Th. 7.2) describes the action of $x_{i}$ in $R^{\lambda}$.

Theorem. The $C l_{k}$-module $R^{\lambda}$ is free with a basis $\left\{v_{\Lambda}: \Lambda \in S T(\lambda)\right\}$ and

$$
x_{p} v_{\Lambda}=c_{\Lambda}(p) v_{\Lambda} \text { for } 1 \leq p \leq k .
$$

\section{Specht MOdules over $H_{k}$}

Let $\lambda$ be a strict partition of $k$ and $\lambda_{n+1}=0$. Define the functional $\lambda$ on the Cartan subalgebra $\mathfrak{h}$ by setting

$$
\lambda\left(E_{i i}\right)=\lambda_{i} ; \quad \lambda\left(F_{i i}\right)=0 .
$$

In $W_{k}=V^{\otimes k}$, where $V$ is the standard $\mathfrak{q}(n)$-module of dimension $(n, n)$, consider the $H_{k}$-submodule $M^{\lambda}$ consisting of the vectors of weight $\lambda$, i.e., $w \in W_{k}$ such that $E_{i i} w=\lambda_{i} w$. Let $\Lambda_{r}$ be a shifted tableau of shape $\lambda$ filled in consecutively along rows from left to right with the numbers 1 to $k$.

Let $\Lambda$ be an arbitrary shifted tableau of shape $\lambda$ and let $R_{\Lambda}$ be the row stabilizer of $\Lambda$. Let $I$ be the sequence obtained by reading the tableau $\Lambda$ from left to right and downwards. For $i \in I$ define

$$
\pi_{i}=\sum_{\alpha \in I, \alpha \text { preceeds } i} \tau_{\alpha, i} .
$$

Set

$$
\kappa_{\Lambda}=\prod_{i \in I}\left(\frac{1}{2} j(j+1)-\pi_{i}^{2}\right),
$$

where $j$ is the number of the column occupied by $i$.

Example 3. For

$$
\Lambda=\begin{array}{llll}
1 & 2 & 4 & 7 \\
& 3 & 5 & 8 \\
& & 6 &
\end{array}
$$

we have

$$
\kappa_{\Lambda}=1 \cdot\left(3-\pi_{2}^{2}\right)\left(3-\pi_{3}^{2}\right)\left(6-\pi_{4}^{2}\right)\left(6-\pi_{5}^{2}\right)\left(6-\pi_{6}^{2}\right)\left(10-\pi_{7}^{2}\right)\left(10-\pi_{8}^{2}\right) .
$$

For every shifted tableau $\Lambda$ of shape $\lambda$ denote by $w_{\Lambda}$ the vector from $W_{k}$ equal to $v_{1} \otimes \cdots \otimes v_{k}$, where $v_{p}=e_{i}$ if $p$ occupies the $i$-th row. Clearly, $w_{\Lambda} \in M^{\lambda}$.

3.1. Theorem. $\kappa_{\Lambda}\left(M^{\lambda}\right)=C l_{k} \kappa_{\Lambda}\left(w_{\Lambda}\right)$ and $\kappa_{\Lambda}\left(w_{\Lambda}\right) \in R^{\lambda}$ is nonzero. 
Proof. Since $M^{\lambda}$ is the module induced from the trivial representation of the row stabilizer of $\Lambda_{r}$, it follows that

$$
\operatorname{Hom}_{H_{k}}\left(T, W_{k}\right)=S^{\lambda_{1}}(V) \otimes \cdots \otimes S^{\lambda_{n}}(V)
$$

where $S^{p}$ is the operator of the $p$-th symmetric power and $V$ is the standard $\mathfrak{q}(n)$ module. Hence, the characteristic of $M^{\lambda}$ is equal to $q_{\lambda_{1}} \ldots q_{\lambda_{n}}$ (see (2.4)). Lemma 2.5 implies that

$$
q_{\lambda_{1}} \ldots q_{\lambda_{n}}=\sum_{\mu \geq \lambda} 2^{l(\lambda)-l(\mu)} m_{\lambda \mu} Q_{\mu} .
$$

Hence, $M^{\lambda}$ is equal to the direct sum of modules isomorphic to either $T^{\mu}$ or $\Pi\left(T^{\mu}\right)$. Further, let $\varphi_{i}: M^{\lambda} \longrightarrow M^{\lambda}$ be a homomorphism such that $\varphi_{i}\left(v_{\Lambda_{r}}\right)=p_{\Lambda_{r}}^{i}$ for $i=1, \ldots, l(\lambda)$, where $p_{\Lambda_{r}}^{i}=\sum_{j \text { lies in the } i \text {-th row }} p_{j}$.

It is not difficult to verify that $\varphi_{1}, \ldots, \varphi_{l(\lambda)}$ generate the Clifford algebra $C l_{l(\lambda)}$. Therefore, $M^{\lambda}$ is equal to the direct sum of modules isomorphic to $R^{\mu}$ (or $\Pi\left(R^{\mu}\right)$ ) for $\mu \geq \lambda$ and the multiplicity of $R^{\lambda}$ in $M^{\lambda}$ is equal to 1 .

It suffices to consider the case when $\Lambda=\Lambda_{c}$ is a shifted tableau of shape $\lambda$ filled from left to right and downwards, as usual. In each module $R^{\mu}$, select a basis $\left\{v_{\Lambda}\right\}$, where $\Lambda$ runs over standard shifted tableaux, as in Theorem 2.6. Let us demonstrate that $\kappa_{\Lambda_{c}}\left(R^{\mu}\right)=0$ if $\mu>\lambda$ and $\kappa_{\Lambda_{c}}\left(R^{\lambda}\right)=C l_{k} v_{\Lambda_{c}}$.

Consider a vector $v_{\Lambda} \in R^{\mu}$ for $\mu \geq \lambda$ and $\Lambda \neq \Lambda_{c}$. There exists a number $j$ such that the $j$-th columns of the tableaux $\Lambda$ and $\Lambda_{c}$ are distinct and all the respective columns with lesser numbers are identical. Since the tableaux are of the same order and $\mu \geq \lambda$, it follows that the length of the $j$-th column of the shifted tableau $\lambda$ is not less than the length of the $j$-th column of the shifted tableaux $\Lambda$. Let $p$ be the least of the numbers occupying the $j$-th column of $\Lambda_{c}$ which is not contained in the $j$-th column of $\Lambda$. Since $\Lambda$ is standard, we see theat $p$ occupies the $(j+1)$-st column and the first row of $\Lambda$.

By Theorem 2.6

$$
\pi_{p}^{2} v_{\Lambda}=\frac{1}{2} x_{p}^{2} v_{\Lambda}=\frac{1}{2}(j+1-1)(j+1-1+1) v_{\Lambda}=\frac{1}{2} j(j+1) v_{\Lambda} .
$$

Hence,

$$
\left(\frac{1}{2} j(j+1)-\pi_{p}^{2}\right) v_{\Lambda}=0 .
$$

Since $\frac{1}{2} j(j+1)-\pi_{p}^{2}$ enters $\kappa_{\Lambda_{c}}$ as a factor, $\kappa_{\Lambda_{c}}\left(v_{\Lambda}\right)=0$. Moreover, since the $\pi_{p}$ for $p=1, \ldots, k$ supercommute with $C l_{k}$, it follows that $\kappa_{\Lambda_{c}}\left(R^{\mu}\right)=0$ if $\mu>\lambda$ and $\kappa_{\Lambda_{c}}\left(v_{\Lambda}\right)=0$ if $v_{\Lambda} \in R^{\lambda}$ and $\Lambda \neq \Lambda_{c}$. Moreover,

$$
\left(\frac{1}{2} j(j+1)-\pi_{p}^{2}\right) v_{\Lambda_{c}}=\left(\frac{1}{2} j(j+1)-\frac{1}{2}(j-i)(j-i+1) v_{\Lambda_{c}}=\frac{1}{2} i(2 j+i-1) v_{\Lambda_{c}},\right.
$$

where $(i, j)$ is the position of $p$. Hence, $\kappa_{\Lambda_{c}}\left(v_{\Lambda_{c}}\right)=\alpha v_{\Lambda_{c}}$ for a nonzero $\alpha$.

Thus, it is shown that $\kappa_{\Lambda_{c}}$ projects $M^{\lambda}$ onto the subspace $C l_{k} v_{\Lambda_{c}}$. To prove the theorem, it suffices to establish that $\kappa_{\Lambda_{c}}\left(w_{\Lambda_{c}}\right) \neq 0$. We induct on $k$. For $k=1$ the statement is obvious. Let $k>1$, let $\Lambda_{c}^{*}$ be obtained from $\Lambda_{c}$ by deleting the last cell in the last column and let $(i, j)$ be the position of $k$. Then

$$
\kappa_{\Lambda_{c}}\left(w_{\Lambda_{c}}\right)=\left(\frac{1}{2} j(j+1)-\pi_{k}^{2}\right) \kappa_{\Lambda_{c}^{*}}\left(w_{\Lambda_{c}^{*}} \otimes e_{i}\right)=\left(\frac{1}{2} j(j+1)-\pi_{k}^{2}\right)\left(\kappa_{\Lambda_{c}^{*}}\left(w_{\Lambda_{c}^{*}}\right) \otimes e_{i}\right) .
$$


By induction, $\kappa_{\Lambda_{c}^{*}}\left(w_{\Lambda_{c}^{*}}\right) \neq 0$ while by Lemma 2.1 and in notations of Lemma 2.1 we have

$$
\left(\frac{1}{2} j(j+1)-\pi_{k}^{2}\right)\left(\kappa_{\Lambda_{c}^{*}}\left(w_{\Lambda_{c}^{*}}\right) \otimes e_{i}\right)=\sum_{m=1}^{n}\left(w_{m} \otimes e_{m}+\bar{w}_{m} \otimes e_{\bar{m}}\right),
$$

where

$$
w_{m}=\left(\frac{1}{2} j(j+1)-\frac{1}{2} E_{i m}^{(2)}-\delta_{i l} \sum_{l=1}^{n} E_{l l}\right) \kappa_{\Lambda_{c}^{*}}\left(w_{\Lambda_{c}^{*}}\right)
$$

and $\bar{w}_{m}=-\frac{1}{2} F_{i m}^{(2)} \kappa_{\Lambda_{c}^{*}}\left(w_{\Lambda_{c}^{*}}\right)$.

It remains to show that $w_{i} \neq 0$. Observe that since $\kappa_{\Lambda_{c}^{*}}\left(w_{\Lambda_{c}^{*}}\right)$ is a highest weight vector with respect to $\mathfrak{q}(n)$, i.e.,

$$
E_{i j} \kappa_{\Lambda_{c}^{*}}\left(w_{\Lambda_{c}^{*}}\right)=F_{i j} \kappa_{\Lambda_{c}^{*}}\left(w_{\Lambda_{c}^{*}}\right)=0 \text { for } i<j,
$$

we have

$$
\begin{aligned}
w_{i} & =\left(\frac{1}{2} j(j+1)-\frac{1}{2} \sum_{r=1}^{n}\left(E_{i r} E_{r i}-F_{i r} F_{r i}\right)-\sum_{r=1}^{n} E_{r r}\right) \kappa_{\Lambda_{c}^{*}}\left(w_{\Lambda_{c}^{*}}\right) \\
& =\left(\frac{1}{2} j(j+1)-\frac{1}{2} \sum_{r>i}^{n}\left(E_{i r} E_{r i}-F_{i r} F_{r i}\right)-\frac{1}{2}\left(E_{i i}^{2}-F_{i i}^{2}\right)-\sum_{r=1}^{n} E_{r r}\right) \kappa_{\Lambda_{c}^{*}}\left(w_{\Lambda_{c}^{*}}\right) \\
& =\left(\frac{1}{2} j(j+1)-\frac{1}{2} \sum_{r>i}\left(\left[E_{i r}, E_{r i}\right]-\left[F_{i r}, F_{r i}\right]\right)\right. \\
& =\left(\frac{1}{2} j(j+1)-\frac{1}{2} \sum_{r>i}^{2}\left(E_{i i}-E_{i i}\right)-\sum_{r=1}^{n} E_{r r}\right) E_{\Lambda_{c}^{*}}\left(w_{\Lambda_{c}^{*}}\right) \\
& \left.=\left(\frac{1}{2} j(j+1)-\frac{1}{2} \sum_{r>i} E_{r r}-\frac{1}{2}\left(E_{i i}^{2}-E_{i i}\right)-E_{i i}\right)-\sum_{r=1}^{n} E_{r r}\right) E_{\Lambda_{c}^{*}}\left(w_{\Lambda_{c}^{*}}\right) \kappa_{\Lambda_{c}^{*}}\left(w_{\Lambda_{c}^{*}}\right) \\
& =\left(\frac{1}{2} j(j+1)-\frac{1}{2} \sum_{r=1} i E_{r r}-\frac{1}{2}\left(E_{i i}^{2}-E_{i i}\right)\right) \kappa_{\Lambda_{c}^{*}}\left(w_{\Lambda_{c}^{*}}\right) \\
& =\left(\frac{1}{2} \lambda_{1}\left(\lambda_{1}+1\right)-\left(\lambda_{1}+\cdots+\lambda_{i}-1\right)-\frac{1}{2}\left(\lambda_{i}-1\right)\left(\lambda_{i}-2\right)\right) \kappa_{\Lambda_{c}^{*}}\left(w_{\Lambda_{c}^{*}}\right) \\
& =\frac{1}{2}\left(\lambda_{1}\left(\lambda_{1}+1\right)-2\left(\lambda_{1}+\cdots+\lambda_{i}\right)+2-\left(\lambda_{i}-1\right)\left(\lambda_{i}-2\right)\right) \kappa_{\Lambda_{c}^{*}}\left(w_{\Lambda_{c}^{*}}\right)
\end{aligned}
$$

If $i$ is the number of the row occupied by $k$, then

$$
\lambda_{1}=\lambda_{i}+i-1, \lambda_{2}=\lambda_{i}+i-2, \ldots, \lambda_{i-1}=\lambda_{i}+1,
$$


and, therefore,

$$
\begin{aligned}
w_{i}= & \frac{1}{2}\left(\left(\lambda_{i}+i-1\right)\left(\lambda_{i}+i\right)-2\left(i \lambda_{i}+1+2+\cdots+i-1\right)\right. \\
& \left.+2-\left(\lambda_{i}-1\right)\left(\lambda_{i}-2\right)\right) \kappa_{\Lambda_{c}^{*}}\left(w_{\Lambda_{c}^{*}}\right)=\lambda_{i} \kappa_{\Lambda_{c}^{*}}\left(w_{\Lambda_{c}^{*}}\right) \neq 0 .
\end{aligned}
$$

3.2. Remark. Observe that

$$
\begin{aligned}
\bar{w}_{i} & =-\frac{1}{2} F_{i i}^{(2)} \kappa_{\Lambda_{c}^{*}}\left(w_{\Lambda_{c}^{*}}\right)=-\frac{1}{2} \sum_{r=1}^{n}\left(F_{i r} E_{r i}-E_{i r} F_{r i}\right) \kappa_{\Lambda_{c}^{*}}\left(w_{\Lambda_{c}^{*}}\right) \\
& =\frac{1}{2} \sum_{i=1}^{n}\left(E_{i r} F_{r i}-F_{i r} E_{r i}\right) \kappa_{\Lambda_{c}^{*}}\left(w_{\Lambda_{c}^{*}}\right)=\frac{1}{2} \sum_{r>i}\left(E_{i r} F_{r i}-F_{i r} E_{r i}\right) \kappa_{\Lambda_{c}^{*}}\left(w_{\Lambda_{c}^{*}}\right) \\
& =\frac{1}{2} \sum_{r>i}\left(\left[E_{i r}, F_{r i}\right]-\left[F_{i r}, E_{r i}\right]\right) \kappa_{\Lambda_{c}^{*}}\left(w_{\Lambda_{c}^{*}}\right)=0 .
\end{aligned}
$$

Hence,

$$
\kappa_{\Lambda_{c}}\left(w_{\Lambda_{c}}\right)=\lambda_{i} \kappa_{\Lambda_{c}^{*}}\left(w_{\Lambda_{c}^{*}}\right) \otimes e_{i}+\sum_{r \neq i}\left(w_{r}+\bar{w}_{r} p_{k}\right) \otimes e_{r}
$$

and by the inductive hypothesis applied to $\kappa_{\Lambda_{c}^{*}}\left(w_{\Lambda_{c}^{*}}\right)$ we deduce that

$$
\kappa_{\Lambda_{c}}\left(w_{\Lambda_{c}}\right)=\lambda_{1} ! \ldots \lambda_{n} ! w_{\Lambda_{c}}+\sum f_{\Lambda} w_{\Lambda} \text {, where } f_{\Lambda} \in C l_{k} \text { and } w_{\Lambda} \neq w_{\Lambda_{c}}
$$

and where the summation runs over the tableaux $\Lambda$ of order $k$ which contain $\lambda_{1}$ symbols 1 or $\overline{1}, \lambda_{2}$ symbols 2 or $\overline{2}$, etc., $\lambda_{n}$ symbols $n$ or $\bar{n}$.

3.3. Specht modules. Recall that for a strict partition $\lambda$ of $k$ the Specht module is the submodule in $M^{\lambda}$ generated by the vectors $\kappa_{\Lambda} w_{\Lambda}$ for all shifted tableaux $s$ of shape $\lambda$.

3.3.1. Theorem. Specht module is equal to $R^{\lambda}$. It is isotypical and its $H_{k}$-centralizer is isomorphic to the Clifford algebra with $l(\lambda)$ generators.

Proof. Recall that $R^{\lambda}$ is the set of highest weight vectors in $W_{k}=V^{\otimes k}$. By the Howe duality between $U(\mathfrak{q}(n))$ and $H_{k}$ the algebra of $H_{k}$-homomorphisms is generated by $U(\mathfrak{q}(n))$. But thanks to Lemma 2.4 we may assume that the algebra of $H_{k}$-homomorphisms is generated by $U(\mathfrak{h})$ for the Cartan subalgebra $\mathfrak{h}$ of $\mathfrak{q}(n)$. If $\lambda_{i}=0$, then, in our notations for the basis of $\mathfrak{h}$, we have

$$
F_{i i}^{2}=E_{i i}=\lambda_{i}=0 \text { in } R^{\lambda} .
$$

But ker $F_{i i}$ is an $\mathfrak{h}$-submodule in the set $\left(V^{\lambda}\right)^{+}$of highest weight vectors. But $\left(V^{\lambda}\right)^{+}$is irreducible as an $\mathfrak{h}$-module by Theorem 2.2. Hence, $\left.F_{i i}\right|_{\left(V^{\lambda}\right)^{+}}=0$ which implies $\left.F_{i i}\right|_{R^{\lambda}}=0$. Thus, the algebra of $H_{k}$-homomorphisms is generated by the $F_{i i}$ for $i$ such that $\lambda_{i} \neq 0$.

By Theorem 3.1 the Specht module is contained in $R^{\lambda}$. Moreover, if $\Lambda$ is a shifted tableau of shape $\lambda$, then

$$
F_{i i} \kappa_{\Lambda} w_{\Lambda}=\kappa_{\Lambda} F_{i i} w_{\Lambda}=\left(\sum_{\alpha \text { lies in the } i \text {-th row of } \Lambda} p_{\alpha}\right) \kappa_{\Lambda} w_{\Lambda} .
$$

Therefore, any homomorphism of $M^{\lambda}$ sends $R^{\lambda}$ into itself. Hence, the Specht module coincides with $R^{\lambda}$. 
3.3.2. Corollary. Let $\Lambda$ be a shifted tableau. Set $p_{\Lambda}^{i}=\sum_{\alpha \text { lies in the } i \text {-th row of } \Lambda} p_{\alpha}$. Then for any $H_{k}$-module endomorphism $\varphi$ of $M^{\lambda}$ we have

$$
\varphi\left(\kappa_{\Lambda} w_{\Lambda}\right)=f \cdot \kappa_{\Lambda} w_{\Lambda},
$$

where $f$ belongs to the subalgebra of $C l_{k}$ generated by $p_{\Lambda}^{1}, \ldots, p_{\Lambda}^{l(\lambda)}$.

Proof. Recall that $M^{\lambda}$ is the subset of vectors of weight $\lambda$ in $W_{k}$. Therefore, any endomorphism of $M^{\lambda}$ may be identified with an element of $U(\mathfrak{q}(n))$; the restriction of this endomorphism onto $R^{\lambda}$ may be identified with an element of $U(\mathfrak{h})$.

But $U(\mathfrak{h})$ is generated by the $F_{i i}$ and to prove the corollary, it suffices to verify it for these elements. We have

$$
F_{i i}\left(\kappa_{\Lambda} w_{\Lambda}\right)=\kappa_{\Lambda}\left(F_{i i} w_{\Lambda}\right)=\kappa_{\Lambda}\left(p_{\Lambda}^{i} w_{\Lambda}\right)=p_{\Lambda}^{i} \kappa_{\Lambda}\left(w_{\Lambda}\right) .
$$

3.3.3. Corollary. Let $b_{\Lambda}$ be the sum of the elements of the row stabilizer of a shifted tableau $\Lambda$ and $\varphi: M^{\lambda} \longrightarrow M^{\lambda}$ be an $H_{k}$-module endomorphism given by the formula $\varphi\left(w_{\Lambda}\right)=b_{\Lambda} \kappa_{\Lambda}\left(w_{\Lambda}\right)$. Then $\left.\varphi\right|_{R^{\lambda}}=c \in \mathbb{C}$.

Proof. Let us show that $\varphi$ commutes with the endomorphisms $F_{i i}$. Indeed,

$$
\begin{aligned}
& \varphi \cdot F_{i i}\left(w_{\Lambda}\right)=\varphi\left(p_{\Lambda}^{i} w_{\Lambda}\right)=p_{\Lambda}^{i} \varphi\left(w_{\Lambda}\right)=p_{\Lambda}^{i} b_{\Lambda} \kappa_{\Lambda}\left(w_{\Lambda}\right) ; \\
& F_{i i} \cdot \varphi\left(w_{\Lambda}\right)=F_{i i} b_{\Lambda} \kappa_{\Lambda}\left(w_{\Lambda}\right)=b_{\Lambda} \kappa_{w}\left(F_{i i} w_{\Lambda}\right) \\
& \quad=b_{\Lambda} \kappa_{\Lambda}\left(p_{\Lambda}^{i} w_{\Lambda}\right)=b_{\Lambda} p_{\Lambda}^{i} \kappa_{\Lambda}\left(w_{\Lambda}\right)=p_{\Lambda}^{i} b_{\Lambda} \kappa_{\Lambda}\left(w_{\Lambda}\right) .
\end{aligned}
$$

The latter identity holds thanks to the fact that $p_{\Lambda}^{i}$ commutes with $b_{\Lambda}$.

Thus, $\varphi \cdot F_{i i}\left(w_{\Lambda}\right)=F_{i i} \cdot \varphi\left(w_{\Lambda}\right)$ and, since the elements $w_{\Lambda}$ generate the $H_{k^{-}}$ module $M^{\lambda}$, we have

$$
\varphi \cdot F_{i i}=F_{i i} \cdot \varphi
$$

Further, $\varphi\left(R^{\lambda}\right) \subset R^{\lambda}$ for any endomorphism $\varphi$ of $M^{\lambda}$, so $\left.\varphi\right|_{R^{\lambda}}$ is an element from the centralizer of $R^{\lambda}$. But by Theorem 3.3.1 the centralizer is the Clifford algebra $C l_{l(\lambda)}$. But $\varphi$ is an even central element of $C l_{l(\lambda)}$, hence, $\varphi$ is a constant.

3.3.4. Corollary. Set $e_{\Lambda}=\kappa_{\Lambda} b_{\Lambda}$. Then

$$
e_{\Lambda}^{2}=c \cdot e_{\Lambda} \text { for } c \in \mathbb{C}, c \neq 0
$$

the algebra $e_{\Lambda} H_{k} e_{\Lambda}$ is isomorphic to $C l_{l(\lambda)}$ and is generated by the $p_{\Lambda}^{i}$ for $1 \leq i \leq$ $l(\lambda)$.

Proof. Thanks to Corollary 3.3.3

$$
\varphi\left(\kappa_{\Lambda}\left(w_{\Lambda}\right)\right)=\kappa_{\Lambda} b_{\Lambda} \kappa_{\Lambda}\left(w_{\Lambda}\right)=c \kappa_{\Lambda}\left(w_{\Lambda}\right)
$$

or, equivalently,

$$
e_{\Lambda}^{2}=c \cdot e_{\Lambda}
$$

If $c=0$, then $\varphi\left(\kappa_{\Lambda}\left(w_{\Lambda}\right)\right)=0$. But the $H_{k}$-submodule generated by $\kappa_{\Lambda}\left(w_{\Lambda}\right)$ coincides with the Specht module, which, thanks to Theorem 3.3.1 is equal to $R^{\lambda}$. So in this case $\varphi\left(R^{\lambda}\right)=0$. On the other hand, Theorem 3.1 implies that $\kappa_{\Lambda}\left(w_{\Lambda}\right)=\alpha w_{\Lambda}+\sum \beta_{T} w_{T}$, where $\alpha \in \mathbb{C}$ and $\beta_{T} \in C l_{k}$ and where $w_{\Lambda} \neq w_{T}$. 
Therefore,

$$
b_{\Lambda} \kappa_{\Lambda}\left(w_{\Lambda}\right)=\alpha \lambda_{1} ! \ldots \lambda_{n} ! w_{\Lambda}+\sum \gamma_{T} w_{T},
$$

where $\gamma_{T} \in C l_{k}$, the sum runs over the tableaux $T$ of order $k$ which contain $\lambda_{1}$ symbols 1 or $\overline{1}, \lambda_{2}$ symbols 2 or $\overline{2}$, etc., $\lambda_{n}$ symbols $n$ or $\bar{n}$, and where $w_{\Lambda} \neq w_{T}$ and $\varphi\left(w_{\Lambda}\right)=b_{\Lambda} \kappa_{\Lambda}\left(w_{\Lambda}\right) \neq 0$. This contradicts the requirement $\varphi\left(R^{\lambda}\right)=0$. So $c \neq 0$.

The algebra $e_{\Lambda} H_{k} e_{\Lambda}$ is anti-isomorphic to the algebra $H_{k}$ of endomorphisms of $R^{\lambda}$ and, thanks to Theorem 3.3.1, it is the Clifford superalgebra $C l_{l(\lambda)}$.

3.3.5. Let $\lambda=(k)$ be the partition consisting of one part, $\Lambda=12 \ldots k$ the corresponding one-row tableau. Then $b_{\Lambda}=\sum_{\sigma \in \mathfrak{S}_{k}} \sigma$ and

$$
\kappa_{\Lambda}=1 \cdot\left(3-\pi_{2}^{2}\right)\left(6-\pi_{3}^{2}\right)\left(10-\pi_{4}^{2}\right) \ldots\left(\frac{1}{2} k(k+1)-\pi_{k}^{2}\right) .
$$

Further, for any $i, j$ and $l$ we have $\left(\tau_{i j}+\tau_{j l}+\tau_{l i}\right) b_{\Lambda}=0$, so

$$
\begin{aligned}
\pi_{p}^{2} b_{\Lambda} & =\left(\sum_{i<p} \tau_{i p}\right)^{2} b_{\Lambda}=\left(p-1-\sum_{i<j<p}\left(\tau_{i p} \tau_{j p}+\tau_{j p} \tau_{i p}\right)\right) b_{\Lambda} \\
& =\left(p-1-\sum\left(\tau_{i j}\left(\tau_{i j}+\tau_{j p}+\tau_{p i}\right)-1\right)\right) b_{\Lambda}=\frac{1}{2} p(p-1) b_{\Lambda} .
\end{aligned}
$$

Therefore, $\kappa_{\Lambda} b_{\Lambda}=k ! b_{\Lambda}=e_{\Lambda}$ and, up to a factor, $e_{\Lambda}$ coincides with the respective Young symmetrizer.

Observe that the symmetrizer Nazarov constructed for the one-row tableau is equal to

$$
\frac{1}{k !} \prod_{j=2}^{k}\left(\prod_{i=1}^{j-1}\left(1+\frac{s_{i j}}{u_{j}-u_{i}}-\frac{p_{i} p_{j} s_{i j}}{u_{j}+u_{i}}\right)\right),
$$

where $u_{p}=\sqrt{p(p-1)}$ and $s_{i j}$ is the transposition; so it does not coincide with $b_{\Lambda}$.

3.3.6. In the case $\lambda=(p+1, p)$ and

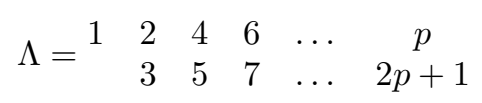

we can simplify the expression for $\kappa_{\Lambda} b_{\Lambda}$. Namely, set

$$
\begin{aligned}
& a_{1}=1+\tau_{12} \tau_{23}+\tau_{23} \tau_{12}, \\
& a_{2}=2+\left(\tau_{14}+\tau_{24}+\tau_{34}\right) \tau_{45}+\tau_{45}\left(\tau_{14}+\tau_{24}+\tau_{34}\right), \\
& a_{3}=3+\pi_{6} \tau_{67}+\tau_{67} \pi_{6}, \\
& \ldots \ldots \ldots \ldots \ldots \ldots \ldots \ldots \ldots \ldots \ldots \ldots \ldots \ldots \ldots \ldots \ldots \ldots \ldots \ldots \ldots \ldots \ldots \ldots \ldots \ldots \ldots \ldots \ldots \\
& a_{p}=p+\pi_{2 p} \tau_{2 p, 2 p+1}+\tau_{2 p, 2 p+1} \pi_{2 p} .
\end{aligned}
$$

Then it is possible to verify that

$$
\kappa_{\Lambda} b_{\Lambda}=a_{1} a_{2} \ldots a_{p} b_{\Lambda}
$$

The author does not know how to simplify the expression for $\kappa_{\Lambda} b_{\Lambda}$ in the general case similarly to the case of the symmetric group; cf. section 1.5 . 


\section{The Specht modules over $\mathfrak{A}_{k}$}

In this section, we construct for $\mathfrak{A}_{k}$ certain analogs of the modules $M^{\lambda}$ and the Specht modules $R^{\lambda}$. First of all, we need the following statement.

4.1. Lemma. Let $\pi_{p}=\sum_{\alpha<p} \tau_{\alpha p}$ for $p=2, \ldots, k$ be odd analogs of Jucys-Murphy's elements. Then for $k \geq 2$

$$
e_{k}=\prod_{p \geq 2} \frac{2}{p(p-1)} \pi_{p}^{2}
$$

is an idempotent and $e_{k} \mathfrak{A}_{k} e_{k}$ is isomorphic to the Clifford algebra $C l_{k-1}$.

Proof. Set $e_{1}=e_{2}=1$. It is easy to verify by induction that for $k \geq 2$ we have

$$
e_{k}=\frac{1}{k !} \sum_{p \geq 1}(-1)^{p} 2^{k-p-1} \Sigma_{2 p+1}
$$

where $\Sigma_{2 p+1}$ is the sum of all elements from $\mathfrak{A}_{k}$ of the form $\tau_{i_{1} i_{2}} \tau_{i_{2} i_{3}} \ldots \tau_{i_{2 \alpha} i_{2 \alpha+1}}$. This implies that $e_{k}$ is a central element that does not vary under automorphisms of $\mathfrak{A}_{k}$ that send $\tau_{i j}$ into $\tau_{\sigma(i) \sigma(j)}$ for any $\sigma \in \mathfrak{S}_{k}$. It is not difficult to verify that

$$
\left(\tau_{12}+\tau_{23}+\tau_{31}\right) \pi_{2}^{2}=0 \text {. }
$$

Hence, $\left(\tau_{12}+\tau_{23}+\tau_{31}\right) e_{k}=0$. Having applied the above described automorphisms to this relation we deduce that

$$
\left(\tau_{i j}+\tau_{i l}+\tau_{l i}\right) e_{k}=0 .
$$

Further, arguments as in section 3.3.5 yield

$$
\pi_{p}^{2} e_{k}=\frac{1}{2} p(p-1) e_{k},
$$

hence,

$$
e_{k}^{2}=\left(\prod_{p \geq 2} \frac{2}{p(p-1)} \pi_{i}^{2}\right) e_{k}=e_{k} .
$$

Furthermore, since $e_{k}$ is a central element, then $e_{k} \mathfrak{A}_{k} e_{k}=\mathfrak{A}_{k} e_{k}$. Let $I$ be the two-sided ideal in $\mathfrak{A}_{k}$ generated by the elements $\tau_{i j}+\tau_{j l}+\tau_{l i}$. Then $e_{k} I=0$. Set $\overline{\mathfrak{A}}=\mathfrak{A}_{k} / I$. In $\overline{\mathfrak{A}}$, then, the following relations hold:

$$
\tau_{12}=\pi_{2}, \tau_{23}=\frac{1}{2}\left(\pi_{3}-\pi_{2}\right), \tau_{34}=\frac{1}{3}\left(\pi_{4}-\pi_{3}\right), \ldots, \tau_{k-1, k}=\frac{1}{k-1}\left(\pi_{k}-\pi_{k-1}\right) .
$$

Hence, $\overline{\mathfrak{A}}$ is generated by the $\pi_{p}$ for $2 \leq p \leq k$. We showed above that condition $\tau_{i j}+\tau_{i l}+\tau_{l i}=0$ in $\overline{\mathfrak{A}}$ implies that $\pi_{i}^{2}=\frac{1}{2}(i-1)(i-2)$ in $\overline{\mathfrak{A}}$. Moreover, $\pi_{i} \pi_{j}+\pi_{j} \pi_{i}=0$ for $i \neq j$. So $\overline{\mathfrak{A}}$ is the Clifford algebra generated by the images of the $\pi_{i}$ for $2 \leq i \leq k$.

Further on, $\left(1-e_{k}\right) I=I$; so $\mathfrak{A}_{k}\left(1-e_{k}\right) \supset I$, and since $\overline{\mathfrak{A}}$ is simple as a superalgebra, we deduce that $\mathfrak{A}_{k}\left(1-e_{k}\right)=I$. Therefore, $\mathfrak{A}_{k} e_{k} \cong \mathfrak{A}_{k} / \mathfrak{A}_{k}\left(1-e_{k}\right) \cong$ $\mathfrak{A}_{k} / I \cong \overline{\mathfrak{A}}_{k}$.

Let $\lambda$ be a strict partition of $k$ and $\Lambda$ a shifted $\lambda$-tableau. Let $\Lambda^{(i)}=\left\{\alpha_{1}, \ldots \alpha_{\lambda_{i}}\right\}$ be the $i$-th row. Recall that

$$
p_{\Lambda}^{i}=\sum_{\alpha \in \Lambda^{(i)}} p_{\alpha}
$$


Let further $\pi_{p}^{(i)}=\sum_{j<p} \tau_{\alpha_{j} \alpha_{p}}$ and

$$
e_{\Lambda}^{i}=\prod_{p=2}^{\lambda_{i}} \frac{2}{p(p-1)}\left(\pi_{p}^{(i)}\right)^{2}, \quad e_{\Lambda}=\prod_{i=1}^{l(\lambda)} e_{\Lambda}^{i} .
$$

Let $V$ be an $(n, n)$-dimensional superspace with $n \geq l(\lambda)$ and $W_{k}=V^{\otimes k}$, where $k=\sum \lambda_{i}$. Let $M^{\Lambda}$ be the subspace of $W_{k}$ spanned by the vectors $w \in W_{k}$ such that

$$
E_{i i} w=\lambda_{i} w ; \quad F_{i i} w=p_{\Lambda}^{i} w \text { for } 1 \leq i \leq l(\lambda) .
$$

Let $R^{\Lambda}=\left\{w \in M^{\Lambda}: E_{i j} w=F_{i j} w=0\right\}$ be the set of highest weight vectors from $M^{\Lambda}$. Recall that the irreducible representations of superalgebra $\mathfrak{A}_{k}$ are labelled by strict partitions $\lambda$ of $k$; moreover, the representation is of type $G$ if $k$ and $l(\lambda)$ are of the same parity, and of type $Q$, otherwise; see [N2].

4.2. Specht modules. Let $\lambda$ be a strict partition of $k$ and $\Lambda$ a shifted tableau of shape $\lambda$. The Specht module for tableau $\Lambda$ is the submodule in $M^{\Lambda}$ generated by the vectors $\kappa_{T} w_{\Lambda}$, where the tableau $T$ is of shape $\lambda$ and its rows have the same numbers as the respective rows of $\Lambda$.

Theorem. i) As $\mathfrak{A}_{k}$-module, $M^{\Lambda}$ is isomorphic to $\mathfrak{A}_{k} e_{\Lambda}$.

ii) The Specht submodule of $M^{\Lambda}$ is equal to $R^{\Lambda}$ and its centralizer is isomorphic to the Clifford superalgebra $C l_{k-l(\lambda)}$.

Proof. i) Let $\mathfrak{A}_{i}$ be the subalgebra of $\mathfrak{A}_{k}$ generated by the $\tau_{\alpha \beta}$, where $\alpha$ and $\beta$ lie in the $i$-th row of $\Lambda$. Define the homomorphism $\varphi_{i}: \mathfrak{A}_{i} \longrightarrow C l_{\lambda_{i}}$ into the Clifford superalgebra on $\lambda_{i}$ generators $p_{\alpha_{1}}, \ldots, p_{\alpha_{\lambda_{i}}}$, where $\alpha_{i}$ runs numbers in the the $i$-th row of $\Lambda$ by setting

$$
\varphi_{i}\left(\tau_{\alpha \beta}\right)=\frac{1}{\sqrt{2}}\left(p_{\alpha}-p_{\beta}\right) .
$$

From Lemma 4.1 we deduce that this homomorphism induces an isomorphism of $\mathfrak{A}_{i} e_{\Lambda}^{i}$ with the subalgebra of $C l_{\lambda_{i}}$ generated by $p_{\alpha}-p_{\beta}$ for $\alpha, \beta \in \Lambda^{(i)}$ (recall that $\Lambda^{(i)}$ is the $i$-th row of $\Lambda$ ).

Therefore, we have an homomorphism

$$
\varphi_{1} \otimes \cdots \otimes \varphi_{l(\lambda)}: \mathfrak{A}_{1} \otimes \cdots \otimes \mathfrak{A}_{l(\lambda)} \longrightarrow C l_{\lambda_{1}} \otimes \cdots \otimes C l_{\lambda_{l(\lambda)}}
$$

which induces an isomorphism of $\mathfrak{A}_{1} \otimes \cdots \otimes \mathfrak{A}_{l(\lambda)} e_{\Lambda}$ with a subalgebra $C l_{\Lambda}$ in $C l_{\lambda_{1}} \otimes \cdots \otimes C l_{\lambda_{l(\lambda)}} \cong C l_{k}$. Moreover, we can see that this subsuperalgebra is generated by the $p_{\alpha}-p_{\beta}$, where $\alpha$ and $\beta$ lie in the same row of $\Lambda$. The dimension of this subalgebra is equal to $2^{\lambda_{1}-1} 2^{\lambda_{2}-1} \ldots 2^{\lambda_{l(\lambda)}-1}=2^{k-l(\lambda)}$. This implies that the ideal $\mathfrak{A}_{k} e_{\Lambda}$ is isomorphic, as an $\mathfrak{A}_{k}$-module, to the module induced from the module $C l_{\Lambda}$ over $\mathfrak{A}_{1} \otimes \cdots \otimes \mathfrak{A}_{l(\lambda)}$, is of dimension

$$
\frac{k !}{\lambda_{1} ! \lambda_{2} ! \ldots \lambda_{l(\lambda)} !} 2^{k-l(\lambda)} \text {. }
$$

It is subject to direct verification that $\operatorname{dim} M^{\Lambda}$ is the same and the homomorphism $\varphi: \mathfrak{A}_{k} e_{\Lambda} \longrightarrow M^{\Lambda}$ such that $\varphi\left(e_{\Lambda}\right)=w_{\Lambda}$ is surjective. Hence, $\varphi$ is an isomorphism. $R^{\Lambda}$.

ii) By Theorem $3.1 \kappa_{T} w_{\Lambda}=\kappa_{T} w_{T} \in R^{\Lambda}$, so the Specht module is contained in 
Now, let us show that the centralizer of $R^{t}$ is isomorphic to the Clifford algebra $C l_{k-l(\lambda)}$.

Obviously, $\mathfrak{A}_{k}$ and $C l_{k} \otimes U(\mathfrak{q}(n))$ form a Howe-dual pair in $W_{k}$. By Theorem 3.3.1 the centralizer of the $H_{k}$-module $R^{\lambda}$ is the Clifford algebra generated by the $F_{i i}$ for $1 \leq i \leq l(\lambda)$. Therefore, the centralizer of the $\mathfrak{A}_{k}$-module $R^{\lambda}$ is the Clifford algebra $C l_{k+l(\lambda)}$ generated by the $F_{i i}$ for $1 \leq i \leq l(\lambda)$ and $p_{1}, \ldots, p_{k}$.

The condition $F_{i i} w=p_{\Lambda}^{i} w$ is equivalent to the condition $e w=w$ for

$$
e=\prod_{1 \leq i \leq l(\lambda)} \frac{1}{2}\left(1-\frac{1}{\lambda_{i}} p_{\Lambda}^{i} F_{i i}\right) \text { and } e^{2}=e .
$$

Therefore, the centralizer of $R^{\Lambda}$ is isomorphic to $e C l_{k+l(\lambda)} e$.

Let $C l_{\Lambda}$ be the subalgebra of $C l_{k}$ generated by the $p_{i}-p_{j}$ for $i, j$ that belong to the same row of $\Lambda$. Then it is not difficult to see that $e C l_{k+l(\lambda)} e \cong C l_{\Lambda}$ and this proves that the centralizer of $R^{\Lambda}$ is isomorphic to $C l_{k-l(\lambda)}$.

Let us prove now that the Specht module is actually equal to $R^{\Lambda}$. Indeed, $C l_{\Lambda}$ naturally acts on $R^{\Lambda}$ by left multiplications and this action commutes with that of $\mathfrak{A}_{k}$. Hence, by the above, any endomorphism of the $\mathfrak{A}_{k}$-module $R^{\Lambda}$ is of the form $w \mapsto f w$, where $w \in R^{\Lambda}$ and $f \in C l_{\Lambda}$, and the algebra of such endomorphisms is generated by multiplications by $p_{\alpha}-p_{\beta}$, where $\alpha$ and $\beta$ lie in the same row of $\Lambda$.

To prove that the Specht module is equal to $R^{\Lambda}$, it suffices to verify that every endomorphism of $R^{\Lambda}$ fixes the Specht module. By the above, it suffices to consider the homomorphism $\varphi$ given by multiplication by $p_{\alpha}-p_{\beta}$, where $\alpha$ and $\beta$ lie in the same row of $\Lambda$. We have

$$
\varphi\left(p_{\alpha}-p_{\beta}\right) \kappa_{T} w_{\Lambda}=\left(p_{\alpha}-p_{\beta}\right) \kappa_{T} w_{\Lambda}=\sqrt{2} \tau_{\alpha \beta} s_{\alpha \beta} \kappa_{T} w_{\Lambda}=\sqrt{2} \tau_{\alpha \beta} \kappa_{s_{\alpha \beta} T} w_{\Lambda} .
$$

But the rows of $s_{\alpha \beta} T$ consist of the same elements that constitute the respective rows of $\Lambda$.

\section{REFERENCES}

[BL] Bernstein J. and Leites D., The superalgebra $Q(n)$, the odd trace and the odd determinant. C.R. Acad. Bulgare Sci. v. 35, no.3, 1982, 285-286. MR 84c:17003

[Ja] James G., The representation theory of the symmetric groups, Lect. Notes Math. 682, 1978. MR 80g:20019

[Jo1] Jones A., The structure of the Young's symmetrizers for spin representations of the symmetric group. I., J. Algebra, 205, 1998, 626-660. MR 99j:20017

[Jo2] Jones A., The structure of the Young's symmetrizers for spin representations of the symmetric group. II., J. Algebra, 213, 1999, 381-404. CMP 99:10

[JN] Jones A. and Nazarov M., Affine Sergeev algebra and $q$-analogs of the Young's symmetrizers for projective representations of the symmetric group, Proc. London Math. Soc., 78, 1999, 481-512. CMP 99:09

[Ju1] Jucys A., Symmetric polynomials and the center of the symmetric group ring, Report Math. Phys. 5, 1974, 107-112. MR 54:7597

[Ju2] Jucys A., Factorization of Young's projection operators for symmetric groups, Litovsk. Fiz. Sb. 5, 1971, 1-10. MR 44:7851

[L] Leites D., Lie superalgebras. In: Modern Problems of Mathematics. Recent developments, v. 25, VINITI, Moscow, 1984, 3-49 (in Russian; the English translation: JOSMAR, v. 30 (6), 1985, 2481-2512). MR 86f:17019

[Ma] Macdonald I., Symmetric functions and Hall polynomials, Oxford Univ. Press, 1995. MR 96h:05207

[Mu] Murphy G., A new construction of the Young seminormal representations of the symmetric group, J. Algebra 69, 1981, 287-291. MR 82h:20014 
[N1] Nazarov M., An orthogonal basis in the irreducible projective representations of the symmetric group, Funct. Anal. Appl., 22, no. 2, 1988, 66-68. MR 89f:20019

[N2] Nazarov M., Young's symmetrizers for projective representations of the symmetric group, Adv. Math., 127, no. 2, 1997, 190-257. MR 98m:20019

[OV] Okunkov A. and Vershik A., A new approach to representation theory of symmetric groups. Selecta Math., new series, 2, no. 4, 1996, 581-605. MR 99g:20024

$[\mathrm{Pe}]$ Penkov I., Characters of typical irreducible representations of finite dimensional $\mathfrak{q}(n)$ modules (Russian), Funktsional. Anal. i Prilozhen. 20, no. 1, 1986, 37-45. MR 87j:17033

[P] Pragacz P., Algebro-geometric applications of Schur $S$ - and $Q$ - polynomials, Lect. Notes Math. 1478, 1991, 130-191. MR 93h:05170

[R] Ram A., Seminormal representations of Weyl groups and Iwahori-Hecke algebras, Proc. London Math. Soc. (3), 75, 1997, 99-133. MR 98d:20007

[Sch] Schepochkina I., Maximal subalgebras of matrix Lie superalgebras, hep-th/9702122.

[S1] Sergeev A., The tensor algebra of the standard representation as a module over the Lie superalgebras $G L(n, m)$ and $Q(n)$, Math. Sbornik, 123 (165), no.3, 1984, 422-430. MR 85h:17010

[S2] Sergeev A., The centre of the enveloping algebra for the Lie superalgebra $Q(n, \mathbf{C})$, Lett. Math. Phys., 7, 1983, 177-179. MR 85i:17004

[S3] Sergeev A., Irreducible representations of solvable Lie superalgebras, math.RT/9810109.

[W] Weyl H., Classical groups, their invariants and representations, Princeton Univ. Press, Princeton, 1939. MR 1:42c

[Ya1] Yamaguchi M., A duality of the twisted group algebra of the symmetric group and a Lie superalgebra, math.RT/9811090.

[Ya2] Yamaguchi M., A duality of the twisted group algebra of the hyperoctaedral group and the queer Lie superalgebra, math.RT/9903159.

On leave of absence from Balakovo Institute of Technique of Technology and Control, Branch of Saratov State Technical University, Russia

Department of Mathematics, University of Stockholm, Roslagsv. 101, Kräftriket hus 6, S-106 91, Stockholm, Sweden

E-mail address: mleites@matematik.su.se (subject: for Sergeev) 